\title{
Identification and characterization of a supraclavicular brown adipose tissue in mice
}

\author{
Qianxing Mo, ${ }^{1}$ Jordan Salley, ${ }^{2}$ Tony Roshan, ${ }^{2}$ Lisa A. Baer, ${ }^{3}$ Francis J. May, ${ }^{3}$ Eric J. Jaehnig, ${ }^{4}$ \\ Adam C. Lehnig, ${ }^{3}$ Xin Guo, ${ }^{2}$ Qiang Tong, ${ }^{2}$ Alli M. Nuotio-Antar, ${ }^{2}$ Farnaz Shamsi, ${ }^{5}$ Yu-Hua Tseng, ${ }^{5}$ \\ Kristin I. Stanford, ${ }^{3}$ and Miao-Hsueh Chen ${ }^{2}$ \\ 'Department of Medicine and Dan L. Duncan Cancer Center, and 'US Department of Agriculture/Agricultural Research \\ Center (USDA/ARS), Children's Nutrition Research Center, Department of Pediatrics, Baylor College of Medicine, Houston, \\ Texas, USA. ${ }^{3}$ Dorothy M. Davis Heart and Lung Research Institute, Department of Physiology and Cell Biology, The Ohio \\ State University Wexner Medical Center, Columbus, Ohio, USA. ${ }^{4}$ Department of Bioengineering, Rice University, Houston, \\ Texas, USA. ${ }^{5}$ Joslin Diabetes Center, Harvard Medical School, Boston, Massachusetts, USA.
}

A fundamental challenge to our understanding of brown adipose tissue (BAT) is the lack of an animal model that faithfully represents human BAT. Such a model is essential for direct assessment of the function and therapeutic potential of BAT depots in humans. In human adults, most of the thermoactive BAT depots are located in the supraclavicular region of the neck, while mouse studies focus on depots located in the interscapular region of the torso. We recently discovered BAT depots that are located in a region analogous to that of human supraclavicular BAT (scBAT). Here, we report that the mouse scBAT depot has morphological characteristics of classical BAT, possesses the potential for high thermogenic activity, and expresses a gene signature that is similar to that of human scBAT. Taken together, our studies reveal a mouse BAT depot that represents human BAT and provides a unique tool for developing new translatable approaches for utilizing human scBAT.

Authorship note: Q. Mo and I. Salley contributed equally to this work.

Conflict of interest: The authors have declared that no conflict of interest exists.

Submitted: January 31, 2017

Accepted: April 20, 2017

Published: June 2, 2017

Reference information: JCI Insight. 2017;2(11):e93166. https:// doi.org/10.1172/jci.insight.93166.

\section{Introduction}

Obesity, a serious health condition arising from the imbalance of energy intake and energy expenditure, is a major risk factor for many life-threatening diseases, including type 2 diabetes and heart disease. The prevalence of obesity and its related comorbidities is alarmingly high, indicating an urgent need for new interventions, such as increasing energy expenditure (1).

One form of energy expenditure, non-shivering thermogenesis, is carried out by thermoactive brown adipocytes within brown adipose tissue (BAT). Brown adipocytes contain multiple small lipid droplets, are densely packed with mitochondria, and express high levels of uncoupling protein 1 (Ucp1) (2-4). Non-shivering thermogenesis is activated by environmental stimuli, such as cold, leading to the increase of sympathetic outflow and the activation of the $\beta 3$-adrenergic signaling in brown adipocytes. This activation then triggers lipolysis, the breakdown of triglycerides in the BAT to free fatty acids by lipases, such as hormone-sensitive lipase ( $H s l)(5)$. BAT can also effectively take up free fatty acids from the bloodstream, a process that largely relies on lipoprotein lipase $(L p l)$ to hydrolyze triglyceride-packed lipoproteins to free fatty acids, which are subsequently internalized through the Cd36 cell surface protein (6). Long-chain fatty acids then activate $U c p 1$ in the mitochondria to generate heat through uncoupling oxidative phosphorylation (7). Brown adipocytes can also actively take up glucose via transporters (Glut4 and Glut1) and utilize it as fuel (7).

The mouse possesses substantial amounts of BAT in both embryos and in adults. Although the precise amounts and anatomical locations of BAT in mouse embryos have not been well documented, studies in adult mice have shown that most of the BAT is located in the dorsal back of the interscapular (interscapular BAT [BAT]) and subscapular regions (subscapular BAT). In addition, small BAT depots are found deep in the dorsal neck between the scapula and the head (cervical BAT), around the aorta within the thoracic cavity (mediastinal BAT), and around the kidney (perirenal BAT) (8-10). iBAT is the most widely used depot for studying BAT function in mice. BAT functions to maintain body temperature and plays a significant role in 
energy expenditure and glucose homeostasis. Recently, another type of thermogenically active adipocyte, the beige adipocyte (also known as the brite adipocyte), was identified within inguinal and epididymal white adipose tissue (iWAT and eWAT) $(11,12)$. Beige adipocytes share many morphological and thermogenic characteristics with brown adipocytes and can become thermoactive after prolonged cold exposure (11).

In contrast to mouse BAT, human BAT is mainly located in the deep neck and clavicular region (1319). The most well-studied human BAT depot is the supraclavicular BAT (scBAT) depot, which is positioned above the clavicle bone $(13,20,21)$. The scBAT depot is larger than the BAT found in the deep neck embedded in the carotid sheath. Some individuals possess additional BAT depots in the axillary, prevertebral regions and kidneys, but scBAT is the BAT depot most consistently found in humans $(16,22)$. The recent recognition of these depots in adult humans has reignited interest in utilizing the metabolic properties of BAT to regulate energy and glucose homeostasis in humans. Most recently, studies have revealed the physiological contributions of human scBAT to whole body energy and glucose homeostasis and identified biomarkers that are specific to human scBAT $(19,23-26)$.

Surprisingly, the two main BAT depots found in adult humans, scBAT and deep neck BAT, have not been reported in mice. Instead, most mouse studies focus on iBAT, which is only present during infancy in humans (22). This discrepancy between mice and humans has cast doubt on how translatable findings in mouse iBAT are to human BAT. To better understand the molecular regulation of human BAT development and function, there is a pressing need to find an animal model that provides an anatomical analog for human scBAT.

To search for mouse BAT depots that resemble human BAT depots, we analyzed mouse embryos and discovered significant amounts of BAT embedded between muscles in the neck (27). In addition to the known dorsal cervical BAT, we identified BAT depots in the ventral site of the neck, including a previously unidentified depot that is the anatomical analog of human scBAT. We named this depot the mouse supraclavicular BAT (scBAT) depot. Here we present, for the first time to our knowledge, a detailed analysis of the anatomical location, morphological characteristics, relative contribution to whole-body glucose homeostasis and thermoregulation, and gene expression profile of this newly identified mouse scBAT.

\section{Results}

Identification of mouse scBAT, a classical BAT depot. We previously performed histological analyses of mouse embryos and found BAT depots in the neck of the mouse embryo (27). In addition to dorsal cervical BAT, we found BAT depots in the ventral neck, where the largest depot was positioned above the clavicle bone and superficial to the sternocleidomastoid muscle, extending from the ventral midline toward the lateral side of neck. Because this location is equivalent to the anatomical location of human supraclavicular BAT, we designated this depot the mouse scBAT depot (Figure 1A). To characterize mouse scBAT, we first performed a detailed histological analysis in late-term mouse embryos (E16.5 to E18.5). At E16.5, scBAT was clearly present in the ventral neck, as shown in transverse sections of the neck. Morphologically, scBAT was composed of islands of brown adipocytes resembling those in iBAT (Supplemental Figure 1A). Examination of the sections revealed that a large portion of the scBAT was located adjacent to the external jugular vein of the neck.

Next, to confirm that the scBAT depot we identified is indeed BAT, we performed immunohistochemistry (IHC) using an antibody against aP2/Fabp4, a fatty acid binding protein expressed in BAT. Our results show that, like iBAT, E16.5 scBAT expressed high levels of aP2 (Supplemental Figure 1B). To determine whether scBAT belongs to the adipose tissue lineage, we performed lineage tracing analysis using aP2-Cre mice bred to Cre-dependent $R O S A^{m T m G}$ reporter mice. In this system, membrane-bound green fluorescent protein (GFP) is activated in the ROSA $A^{m T m G}$ reporter mouse after aP2-Cre-mediated excision. Like iBAT, scBAT is strongly labeled by GFP in aP2-Cre; ROS $A^{m T m G}$ mouse embryos, indicating that brown adipocytes originate from a lineage expressing aP2 (Supplemental Figure 1C). By E18.5, a substantial portion of the ventral neck contained scBAT. At this stage, scBAT was more visible in transverse sections of the mouse neck and could be easily distinguished from the dorsal cervical BAT (cBAT) and iBAT depots (Figure 1B). The depot was also more accessible at this stage and could be isolated from the mouse.

Last, we removed the scBAT and iBAT from E18.5 embryos and performed quantitative RT-PCR (qRT-PCR) to determine whether the gene expression profile of mouse scBAT was similar to that of iBAT. We measured genes that regulate brown adipogenesis, including Pparg2, Cebpa, Cebpb, Ebf2, $\operatorname{Prdm16}$, and $Z f p 423$, and genes involved in lipid accumulation and glucose uptake, including aP2, Adipoq, and Glut4, and thermogenesis, including Ucp1, Pgc1a, and Dio2 (28). These BAT-specific genes were expressed in embryonic scBAT at levels similar to those in embryonic iBAT (Figure 1C). In addition, the 
A

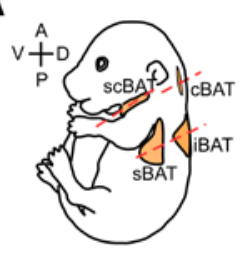

C

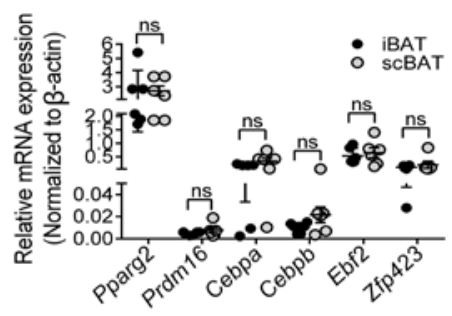

B

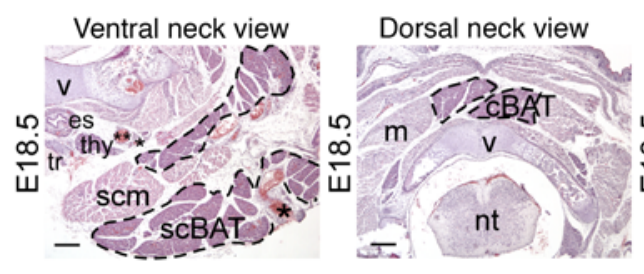

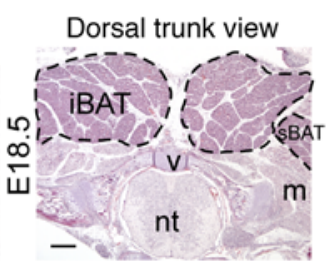

D

IBAT $\mathrm{SCBAT}$
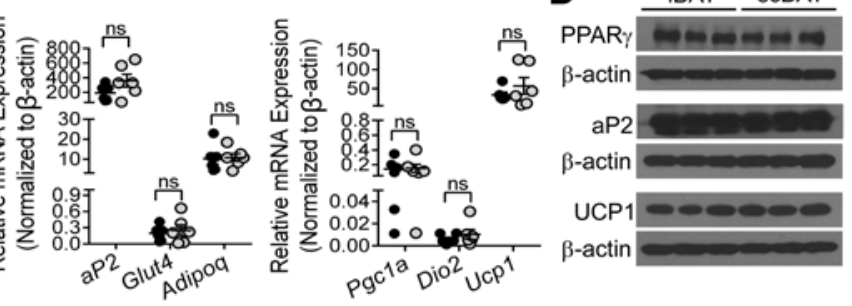

Figure 1. Identification of a supraclavicular BAT (scBAT) depot that forms during development in mice. (A) Diagram of a E18.5 mouse embryo showing the positions of supraclavicular (scBAT), cervical (cBAT), interscapular (iBAT), and subscapuIar (sBAT) BAT depots. A, anterior; P, posterior; V, ventral; D, dorsal. Dotted lines indicate the planes of sections presented in B. (B) Representative H\&E-stained histological sections (transverse sections) from E18.5 mouse embryo neck and thoracic regions showing scBAT, cBAT, iBAT, and part of sBAT depots. Scale bars: $200 \mu \mathrm{m}$. v, vertebrate; es, esophagus; thy, thyroid; tr, trachea; scm, sternocleidomastoid; m, muscle; nt, neural tube. ${ }^{*}$ External and internal jugular veins. ${ }^{*}$ Carotid artery. (C) Relative mRNA expression of BAT markers in iBAT and scBAT isolated from E18.5 mouse embryos. Data are presented as mean \pm SEM. $n=6$. ns, nonsignificant $(P>0.05)$. Two-tailed $t$ test. (D) Western blots of BAT selective markers in iBAT and scBAT isolated from E18.5 mouse embryos. $n=3$. $\beta$-Actin was used as loading control.

protein levels of key BAT regulators (PPAR $\gamma, \mathrm{aP} 2$, and $\mathrm{UCP} 1)$ were similar in embryonic scBAT and iBAT (Figure 1D). Taken together, these histological, lineage, and molecular analyses suggest that scBAT is a previously uncharacterized BAT depot in the embryonic mouse neck.

Mouse scBAT continues to develop after birth. Up to this point, to our knowledge, the only BAT depot that has been identified in the adult mouse neck is cervical BAT (cBAT). Since we identified a depot distinct from cBAT in mouse embryos, we sought to determine whether scBAT was present in the adult mouse neck. Mice were dissected at 3, 8, 24-26, and 52 weeks of age. At 3 weeks of age (weaning), scBAT could be seen in the ventral neck (Supplemental Figure 2A). To verify that scBAT is BAT not WAT, we isolated and compared scBAT, iBAT, and iWAT. The isolated scBAT was more similar morphologically to iBAT than iWAT (Supplemental Figure 2B), indicating that, like iBAT, scBAT is classical BAT. We also performed IHC using PPAR $\gamma$ and UCP1 antibodies to confirm that scBAT was indeed BAT (Supplemental Figure 2C). At 8 weeks of age (young adult), scBAT could be clearly identified in the ventral neck of mice and was tightly connected to the jugular veins, specifically, the external jugular vein (Figure 2, A-C, and Supplemental Figure 3, A-E). The same scBAT depot could also be seen in older mice at 24 and 52 weeks of age (Figure 2B).

The general appearance of scBAT was different from iBAT. iBAT was a thick tissue composed of tightly connected small brown adipocyte lobes, while scBAT was shaped like a thin strip of loosely connected small brown adipocyte lobes (Figure 2D and Supplemental Figure 3, F and G). Furthermore, the absolute and normalized weights of scBAT were much smaller than the weights of iBAT ( $40 \%-50 \%$ of iBAT) at all postnatal stages analyzed (Figure 2E). Interestingly, neither iBAT nor scBAT increased in mass between 3 and 8 weeks of age (Figure 2E). As mice aged from 8 to 52 weeks of age, the BAT mass increased, but a much larger increase in BAT mass was observed for iBAT than for scBAT. To compare the morphology of scBAT to that of other adipose tissue depots in adult mice, we performed H\&E staining of iBAT, scBAT, iWAT, and eWAT. We found that scBAT in the adult mouse morphologically resembled iBAT and contained multiple small lipid droplets (Figure 2, F and G). To compare the genetic profile of scBAT with that of other adipose tissue depots, we conducted gene expression analyses to measure the expression of a panel of selected adipose tissue and BAT markers in iBAT, scBAT, iWAT, and eWAT. Pparg2, Cebpa, Ceb$p b$, Prdm16, Ebf2, aP2, and Adipoq were expressed in all 4 of these depots, and the BAT marker Cidea was expressed in scBAT at levels similar to those in $\mathrm{BATT}$ (Figure $2 \mathrm{H}$ ). Thus, these data provide evidence of the existence of a scBAT depot in adult mice. 
The thermogenic potential of scBAT in adult mice is similar to that of iBAT. After confirming that adult mice possess scBAT, we investigated whether its potential for thermogenesis is similar to that of iBAT. Thermogenesis occurs within the mitochondria, so we first determined the amount of mitochondria in scBAT. We isolated iBAT, scBAT, iWAT, and eWAT from 8-week-old adult mice and performed transmission electron microscopy (TEM) studies. Similar to iBAT, scBAT was densely packed with mitochondria, while only trace amounts of mitochondria were present in WAT (Figure 3, A and B). qRT-PCR showed that mitochondrial components (Cycs [cytochrome $c$ ], Cox5b, Cox7a1, and Cox8b) were also significantly increased in iBAT and scBAT compared with WAT (Figure 3C).

Next, to determine the levels of the major mediators of the thermogenesis in scBAT, we examined the expression of uncoupling protein family members (Ucp1, Ucp2, and $U_{c p} 3$ ). $U_{c p} 1$ was highly expressed in scBAT, while $U c p 2$ and $U_{c p} 3$ were expressed at much lower levels (Figure 3D and Supplemental Figure 4). Ucp1 was expressed at similar levels in both scBAT and iBAT, levels that were much higher than those in iWAT (Figure 3D). Other thermogenic genes, Dio2 and Pgcla, were also expressed in scBAT at levels comparable to those in iBAT (Figure 3D). Mitochondrial component proteins COXIV and UCP1 were also abundant in iBAT and scBAT (Figure 3E). These results indicate that scBAT possesses the molecular machinery and, thus, high potential for thermogenic activation and provide strong evidence that scBAT is a classical BAT depot.

The $\beta 3$-adrenergic signaling pathway induces the expression of genes associated with activated BAT in scBAT in vivo. Activation of thermogenesis in BAT (specifically iBAT) depends on $\beta$-adrenergic signaling via the $\beta 3$-adrenergic receptor (Adrb3) (7). To determine whether scBAT has the potential to be activated through this mechanism, we measured expression of $\beta$-adrenergic receptors (Adrb1, Adrb2, and Adrb3) in scBAT by qRT-PCR analyses. Similar to iBAT, scBAT showed high expression of Adrb3 and lower expression of $A d r b 1$ and Adrb2 (Figure 3F). Since Adrb3 is the main adrenergic receptor expressed in scBAT, we tested whether scBAT could be activated in vivo by injection of the Adrb3 selective agonist CL316243. After injection, we measured the expression of $\mathrm{Hsl}$, the enzyme that breaks down intracellular triglycerides, as well as $L p L$ and $C d 36$, regulators of fatty acid uptake from the pool of triglycerides in the circulation. The expression of $H s l$ increased after CL316243 treatment in both iBAT and scBAT, indicating the potential for CL316243 to trigger lipolysis in scBAT (Figure 4A). Interestingly, Hsl expression was actually higher in scBAT than in iBAT after 3 days of CL316243 treatment. $\mathrm{Lpl}$ and Cd36 expression also increased in scBAT after injection of CL316243 (Figure 4A). CL316243 injection also increased expression of Glut4 and of the BAT-specific genes Pparg2, Cidea, and $U c p 1$ (Figure 4A). UCP1 protein levels also increased after CL316243 treatment (Figure 4B). These data indicate that $\beta 3$-adrengeric stimulation increases expression of the machinery required for uptake of free fatty acids and glucose, breakdown of triglycerides, and activation of thermogenesis in scBAT.

Transplantation of ScBAT improves glucose homeostasis and thermoregulation in mice. To determine whether scBAT improves glucose metabolism in vivo, we utilized a transplantation model. Previous studies have shown that transplantation of iBAT into adult mice significantly improved glucose homeostasis and thermoregulation, demonstrating the beneficial metabolic effects of iBAT $(29,30)$. Here, we examined whether transplantation of scBAT into healthy adult mice has the same effect as transplantation of iBAT. We isolated both scBAT and iBAT from 12-week-old C57BL/6 male mice and transplanted the tissues into the visceral cavity of age-matched adult male mice. A total of 0.1 $\mathrm{g}$ of scBAT pooled from 3 mice and $0.1 \mathrm{~g}$ of iBAT from 1 mouse were used for the transplantation. Sham-operated mice were used as controls. Transplantation of both scBAT and iBAT improved glucose tolerance compared with sham-operated mice 10-12 weeks after transplantation (Figure 5, A and B). Insulin tolerance tests (ITTs) indicated that mice transplanted with either scBAT or iBAT were more insulin sensitive than sham-operated mice 12 weeks after transplantation (Figure 5C). Mice receiving $\mathrm{BBAT}$ had a greater improvement in glucose tolerance tests (GTTs) compared with mice transplanted with scBAT, but transplantation of either scBAT or iBAT improved ITTs to a similar extent. While we observed a significant improvement in glucose homeostasis after BAT transplantation, we did not observe any changes in body weight in iBAT- and scBAT-transplanted mice during the 12 weeks after transplantation (Supplemental Figure 5A). Interestingly, the endogenous mass of both iBAT and scBAT increased in mice receiving transplants, with the most notable increase being in the endogenous mass of scBAT in mice transplanted with scBAT (Supplemental Figure 5B).

Since BAT is a thermogenic tissue, we performed cold-exposure studies to determine whether the transplanted BAT maintained thermogenic properties. Mice receiving BAT did not differ in basal body temperature from sham-operated mice, but they had a strikingly better ability to maintain body 
A

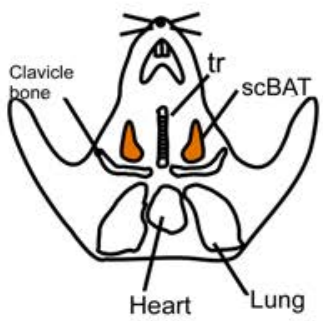

D
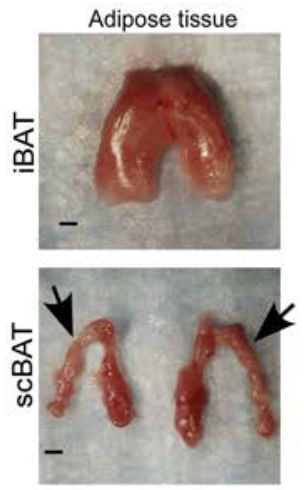

8-week-old

B
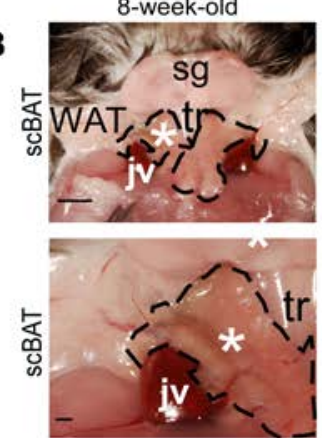

24-week-old
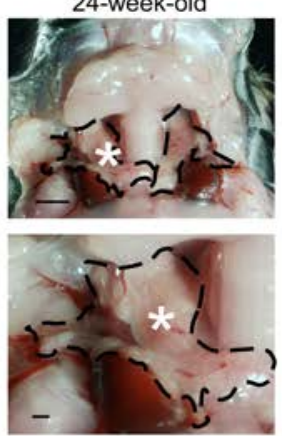

52-week-old
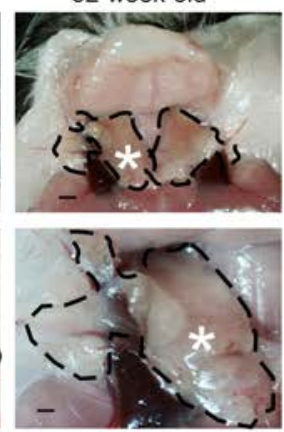

8-week-old
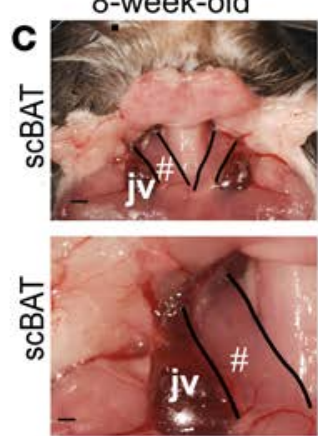

E
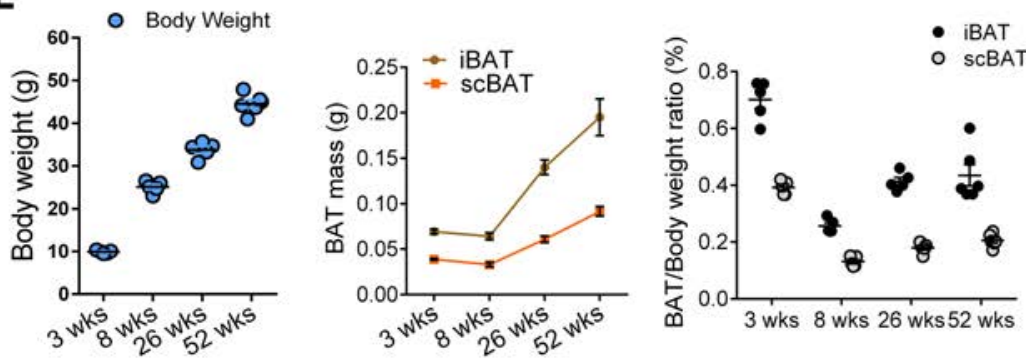

$\mathbf{F}$<smiles>[CH2-][13CH3]</smiles>

$\underset{\mathrm{x}}{\mathrm{x}}$

iBAT

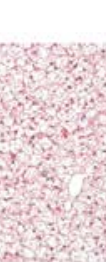

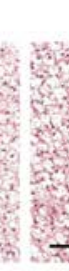

ScBAT

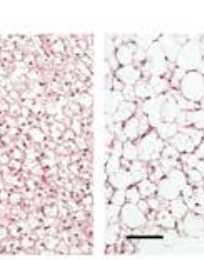

IWAT

INAT

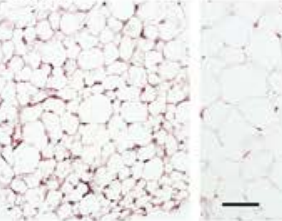

eWAT

\section{G}

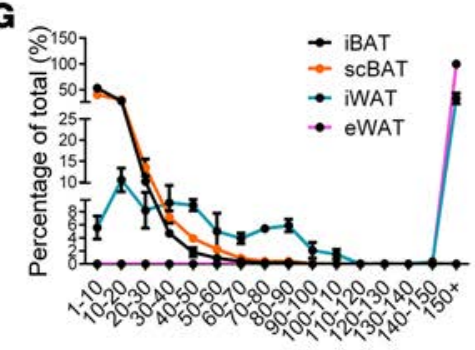

Lipid droplets $\left(\operatorname{area}, \mu \mathrm{m}^{2}\right)$

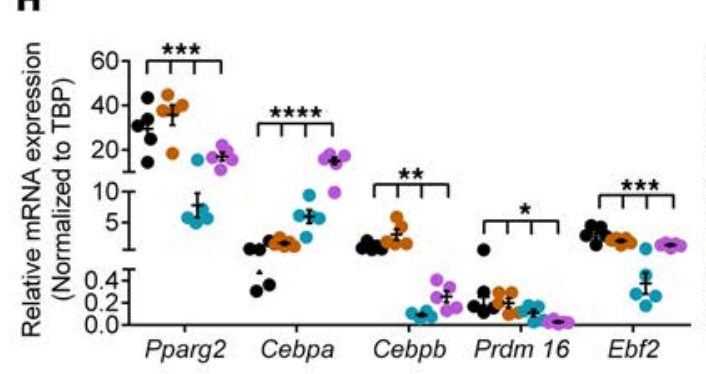

- iBAT • scBAT • iWAT - eWAT

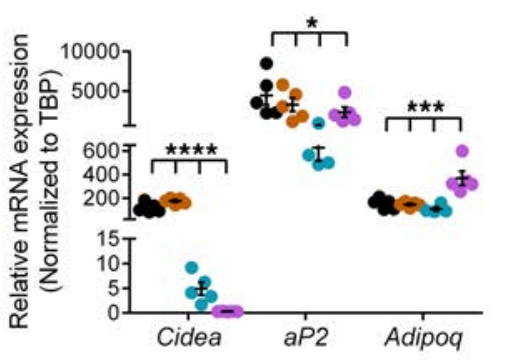

- IBAT • SCBAT • IWAT • eWAT

Figure 2. Mouse scBAT continues to grow after birth. (A) Diagram showing the anatomical location of scBAT in adult mice (ventral view). (B) Representative images showing the anatomical location of scBAT in 8-, 24-, and 52-week-old mice. Top row: Lower-magnification images of the ventral neck. sg, salivary gland; tr, trachea; jv, external jugular vein. scBAT is outlined by the black dotted line. Bottom row: Higher-magnification images of the ventral neck showing close-up images of scBAT. * scBAT. Scale bars: 1,000 $\mu \mathrm{m}$. (C) Representative image of the ventral neck from an 8-week-old mouse showing the sternocleidomastoid muscle (\#) after scBAT was removed. Scale bars: 1,000 $\mu \mathrm{m}$. (D) Representative images of iBAT and scBAT isolated from an 8-weekold mouse. Arrows point to the part of the scBAT that is located behind the external jugular vein. Scale bars: 1,000 $\mu$ m. (E) Body weight, iBAT and scBAT mass, and weight/mass ratio of iBAT and scBAT in 3-, 8-, 26-, and 52-week-old male mice. $n=5-6$. (F) Representative H\&E-stained sections of iBAT, SCBAT, iWAT (inguinal WAT), and eWAT (epididymal WAT) isolated from 8-week-old male mouse. $n=3$. Scale bars: $50 \mu \mathrm{m}$. (C) Lipid droplet size distribution in iBAT, scBAT, iWAT, and eWAT from 8-week-old male mice. Percentages for different lipid droplet sizes were measured in 4-8 randomly selected H\&E-stained sections from 3 mice. (H) Relative mRNA expression of BAT selective markers in iBAT, scBAT, iWAT, and eWAT isolated from 8-week-old male mice. Data are presented as mean \pm SEM. $n=4-5$. ${ }^{* * *} P<0.0001,{ }^{* * *} P<0.001,{ }^{* *} P<0.01,{ }^{*} P<0.05$. One-way ANOVA. 
A

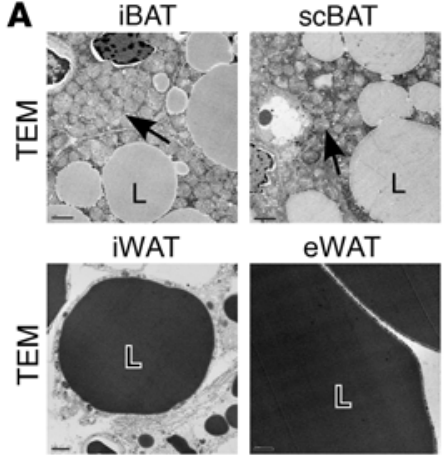

D

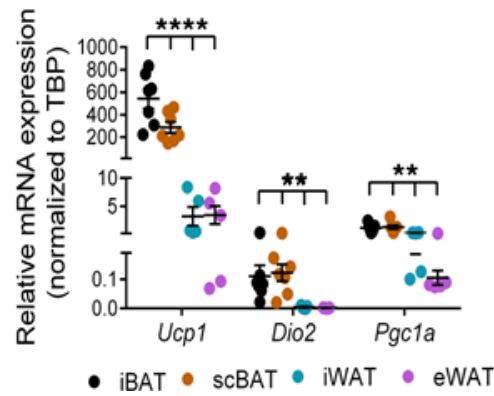

B

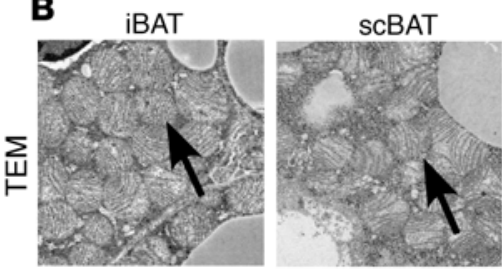

C

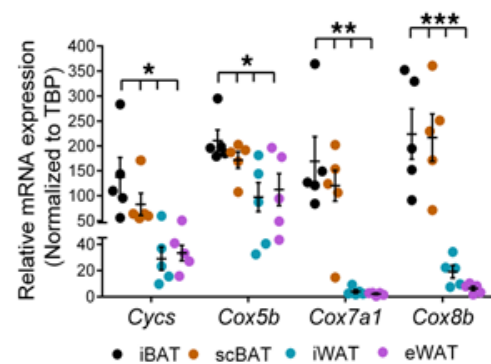

E
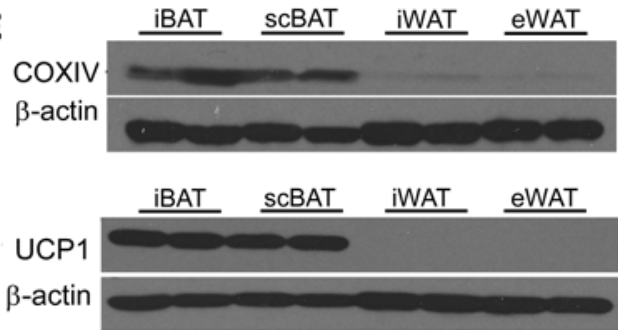

F

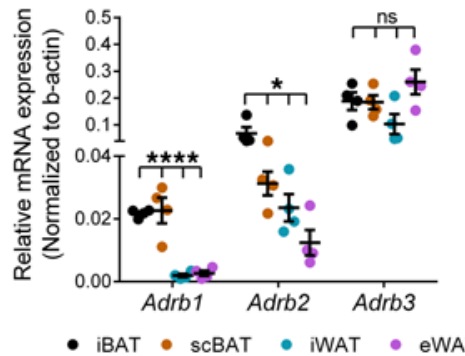

Figure 3. scBAT contains a high density of mitochondria and expresses thermogenic regulators at levels comparable to iBAT. (A) Representative transmission electron micrograph (TEM) images showing mitochondria density in iBAT, scBAT, iWAT, and eWAT from 8-week-old mice. $n=3$. Scale bars: 1,000-1,200 $\mu \mathrm{m}$. L, lipid droplet. Lipids were stained with osmium tetroxide, and the staining was slightly stronger in WAT (dark gray) than in BAT (light gray). Arrows point to mitochondria. (B) Cropped images of iBAT and scBAT shown in Figure 3A. Arrows point to mitochondria. (C) Relative mRNA expression of mitochondrial biogenesis markers in iBAT, scBAT, iWAT, and eWAT from 8-week-old mice. Data are presented as mean $\pm \mathrm{SEM} . n=5$. ${ }^{* * *} P<0.001,{ }^{* *} P<0.01$, ${ }^{*} P<0.05$. Oneway ANOVA. (D) Relative mRNA expression of thermogenesis markers in iBAT, scBAT, iWAT, and eWAT from 8-week-old mice. Data are presented as mean \pm SEM. $n=5-7 .{ }^{* * *} P<0.0001,{ }^{* *} P<0.01$. One-way ANOVA. (E) Representative Western blot images of COXIV and UCP1 in adipose tissues from 8-week-old mice. $\beta$-Actin was used as protein loading control. $n=2$. (F) Relative mRNA expression of adrenergic receptors in iBAT, scBAT, iWAT, and eWAT from 8-weekold male mice. Data are presented as mean \pm SEM. $n=4$. ${ }^{* * *} P<0.0001,{ }^{*} P<0.05$; ns, nonsignificant $(P>0.05)$. One-way ANOVA.

temperature when exposed to cold $\left(4^{\circ} \mathrm{C}\right)$ (Figure $5 \mathrm{D}$ ), suggesting that transplantation of BAT enhances thermogenic activity in these mice (Figure 5D). These results demonstrate that transplanted scBAT improves glucose homeostasis and thermogenesis to a similar extent as transplanted iBAT.

Global analysis of gene expression in the scBAT of adult mice. Our data demonstrate that mouse scBAT is a classical BAT depot. However, because there are distinct differences between iBAT and scBAT, we investigated whether scBAT has a specific unique molecular signature. We first examined by qRT-PCR the expression of several molecular markers for adipose tissue in scBAT, iBAT, iWAT, and eWAT isolated from 8-week-old mice. WAT-specific markers $\operatorname{Hox} C 8$ and $\operatorname{Hox} C 9(31,32)$ were highly expressed in all WAT depots but not in iBAT or scBAT depots (Figure 6A). The BAT-specific marker Zic1 (32) was highly expressed in iBAT but was expressed at very low levels in scBAT, iWAT, and eWAT (Figure 6A). In contrast, another BAT marker, Lhx8, was highly expressed in iBAT as well as in scBAT and iWAT, suggesting that $L h x 8$ is broadly expressed in both BAT and WAT (Figure 6A). Last, we measured the expression of 3 widely used beige adipocyte markers, Tbx1, Cd137 (also known as Tnfrsf 9 ) (11), and Cited1 (33). Previous studies showed that expression of these 3 markers is specific to beige adipocytes residing within iWAT $(11,33)$. We found that $T b x 1$ and Cited 1 were both highly expressed in iWAT and expressed at lower levels in iBAT, while Cd137 was expressed at various levels in both BAT and WAT. Intriguingly, we also found that both $T b x 1$ and Cited 1 were expressed in scBAT (Figure 6A), suggesting that these markers are unable to distinguish scBAT from iWAT. Altogether, our results suggest that the markers currently employed to identify various adipose tissues might not be as specific as previously indicated and that there is a need to identify markers that more precisely define mouse scBAT.

To identify scBAT-specific markers, we performed RNA-sequencing analyses on total RNA isolated from iBAT, scBAT, iWAT, and eWAT from 8-week-old mice (Figure 6B). Because our goal was to identify scBAT-specific genes, these mice were housed at $22^{\circ} \mathrm{C}$ to minimize substantial activation of beige adipocytes by cold stimulation in iWAT. We chose to use adipose tissue depots as our starting material 

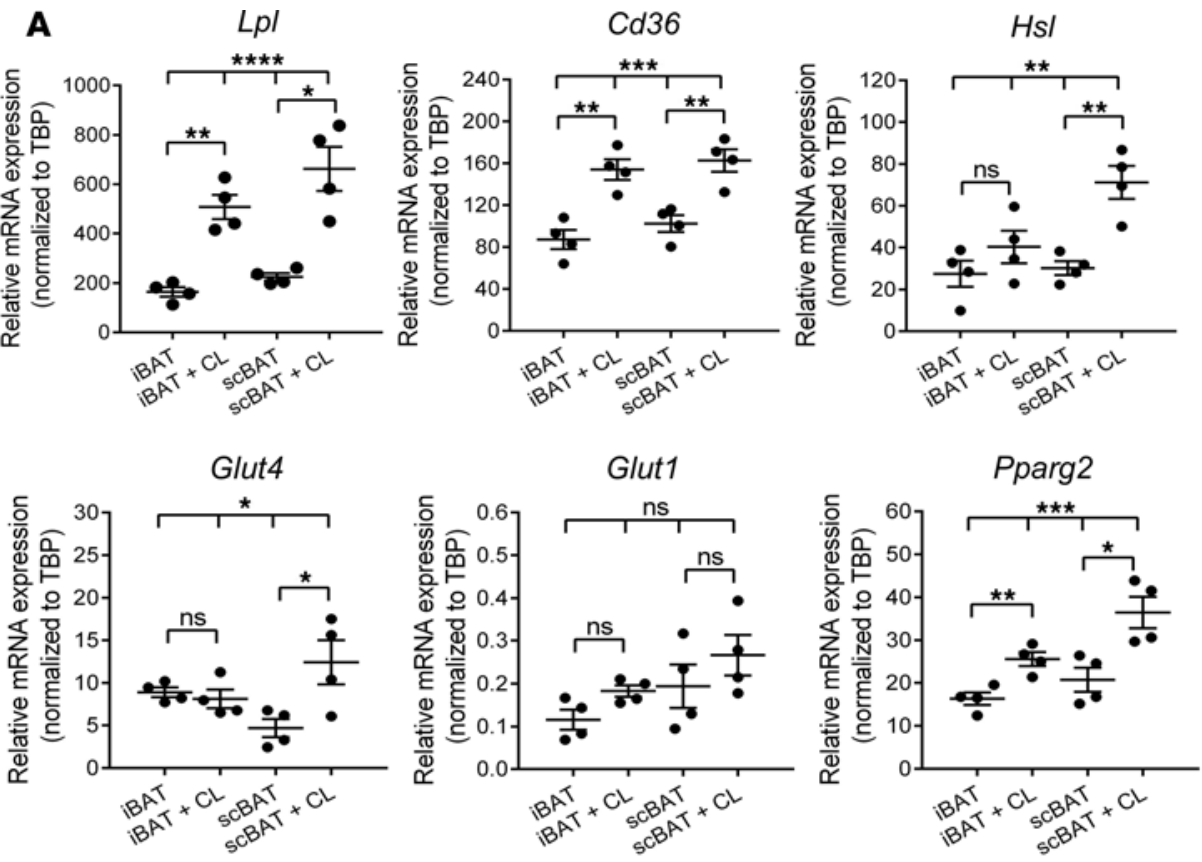

$\operatorname{Prdm16}$

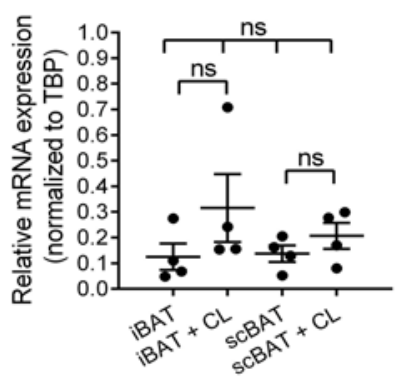

Cidea
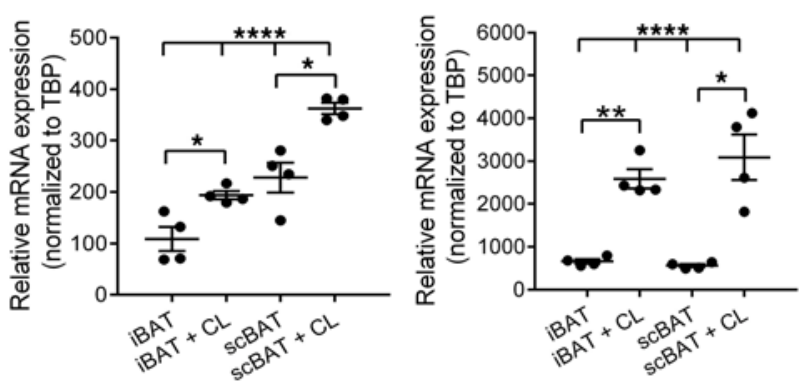

Pgc1a
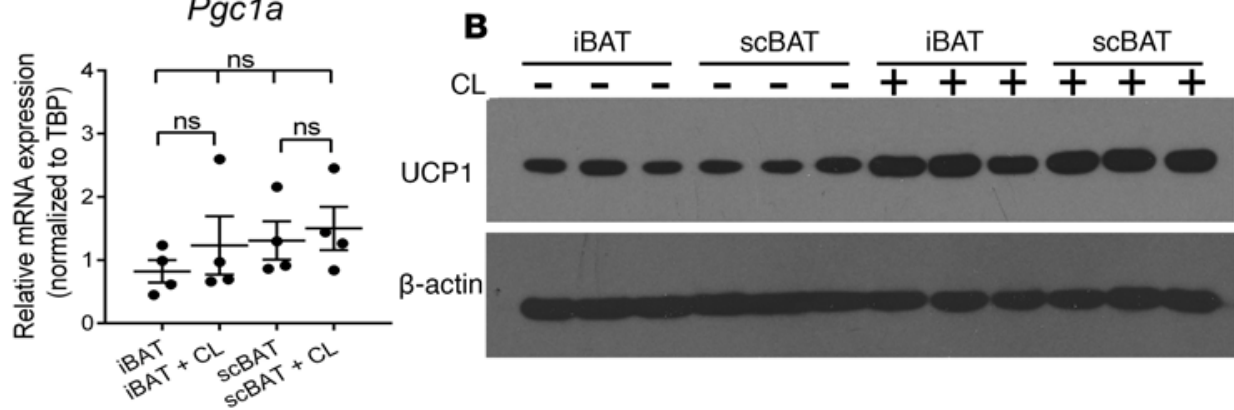

Figure 4. The $\boldsymbol{\beta} 3$-adrenergic pathway mediates thermoactivation of scBAT in mice. (A) Effect of CL316243 (CL) treatment on relative expression of lipid, glucose uptake, and thermogenesis marker mRNA in iBAT and scBAT in 12-weekold mice. Data are presented as mean \pm SEM. $n=4$. ${ }^{* * *} P<0.0001,{ }^{* * *} P<0.001,{ }^{* *} P<0.01,{ }^{*} P<0.05$; ns, nonsignificant $(P>0.05)$. Two-tailed $t$ test for 2 groups and 1-way ANOVA for 4 groups. (B) Western blot image of UCP1 in iBAT and scBAT from 12-week-old mice treated with or without CL316243. $\beta$-Actin was used as protein loading control. $n=3$.

rather than cultured preadipocytes to ensure that we obtained complete profiles of gene expression for the depots rather than for subsets of cells in the depots. Our RNA-sequencing results indicated that BAT-specific genes (Ebf2 and Prdm16) and thermogenic and mitochondrial biogenesis genes (Ucp1, Dio2, Pgc1a, Cox7a1, and Cox8b) were differentially expressed in both iBAT and scBAT compared with WAT depots (Figure 6C), supporting the qRT-PCR analyses of scBAT presented in Figures 2 and 3. These sequencing data also support our qRT-PCR observations (Figure 6A) that $L h x 8$ and $T b x 1$ are not specific markers for classical BAT $(L h x 8)$ and beige adipocytes (Tbx1) (Figure 6C). 

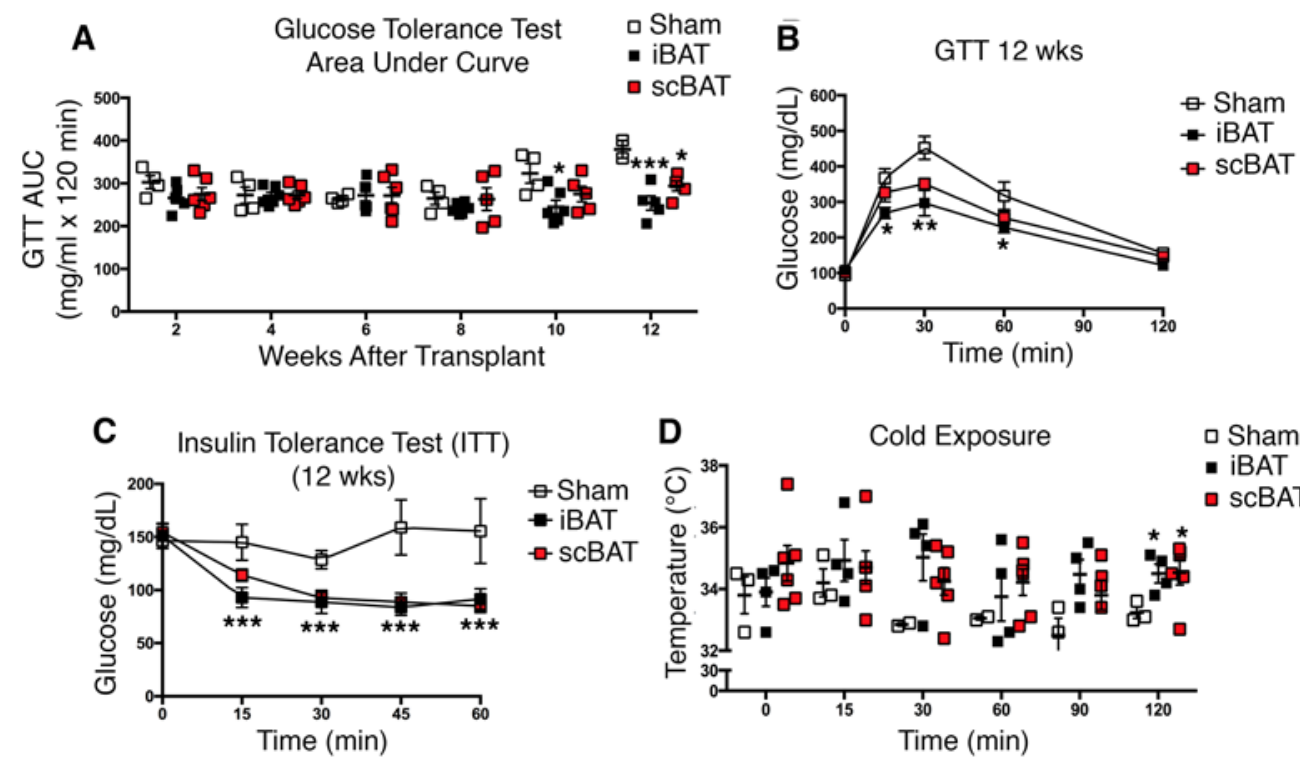

Figure 5. scBAT transplantation improves glucose tolerance, insulin sensitivity, and thermoregulation. In vivo metabolic and thermogenic analyses of iBAT and scBAT were performed on 12-week-old male mice receiving transplants of $0.1 \mathrm{~g}$ iBAT, $0.1 \mathrm{~g}$ scBAT, or no BAT (sham-operated). (A) Glucose tolerance test (CTT) area under the curve (AUC). GTTs were performed every 2 weeks after transplantation. Data are presented as mean \pm SEM. $n=4-6$ per group. ${ }^{* *} P<0.001,{ }^{*} P<0.05$ between BAT transplanted group and control group. (B) GTT curves. GTTs were performed at 12 weeks after transplantation. $n=4-6$ per group. ${ }^{*} P<0.01,{ }^{*} P<0.05$. (C) Insulin tolerance test (ITT) curves. ITTs were performed at 12 weeks after transplantation. $n=4-6$ per group. ${ }^{* *} P<0.001$. (D) Body temperature in mice exposed to cold $\left(4^{\circ} \mathrm{C}\right)$ for the indicated times. Short-term cold exposure experiments were performed on iBAT- and scBAT-transplanted mice at 12 weeks after transplantation. $n=3-6$ per group. ${ }^{*} P<0.05$ between BAT-transplanted group and control group. One-way ANOVA.

To determine whether the overall expression profile of scBAT resembles that of BAT or WAT, we performed principal component analysis and found that scBAT clustered more closely to iBAT than to iWAT and eWAT (Figure 6D). This data is also supported by gene ontology (GO) analysis showing that the set of genes expressed at higher levels in scBAT than in iWAT was enriched for genes involved in thermogenic and metabolic pathways, including glucose and lipid metabolic processes, acetyl-CoA biogenesis, brown fat cell differentiation, and energy reserve metabolic process (Supplemental Table 1). Taken together, these data indicate that scBAT expresses genes associated with classical BAT function even in the absence of cold exposure.

Last, our analysis also identified 61 genes that were expressed at a significantly higher level (at least 5 -fold) in scBAT than in both iBAT and WAT (Figure 6E and Supplemental Table 2). Among these 61 genes, we randomly selected 10 genes to validate their expression in iBAT, scBAT, iWAT, and eWAT isolated from 8-week-old mice. We found 3 genes that showed higher expression in scBAT than other depots: Lhx1 (LIM homeobox protein 1), Bglap2 (bone gamma-carboxyglutamate protein 2, also known as osteocalcin), and Cst10 (cystatin 10) (Figure 7, A and B). We also found that Lhx1 and Bglap2 were expressed in an immortalized brown preadipocyte cell line derived from mouse scBAT (P7scBAT-C4 cells) (Supplemental Figure 6, A and B). After differentiation was induced, Lhx1 transcripts increased and Bglap2 transcripts decreased (Figure 7C), while Cst10 was not expressed in either preadipocytes or mature adipocytes.

Genes from the molecular signature of human scBAT are expressed in mouse scBAT. To determine whether the molecular signature of mouse scBAT is similar to that of human scBAT, we measured expression of genes from the known molecular signature of human scBAT in mouse scBAT, specifically Kcnk3 and Mtus1, which were identified in immortalized human supraclavicular brown preadipocyte cell lines (26). Knck3 was expressed at higher levels in mouse iBAT and scBAT than in iWAT or eWAT (Figure 7D), and Mtusl expression was slightly higher in mouse scBAT than in the other depots (Figure 7D). As in studies using human supraclavicular brown adipocytes (26), we also found that both Kcnk3 and Mtus1 were expressed at much higher levels in mature P7scBAT-C4 adipocytes compared with preadipocytes (Figure 7E). In addition, two markers that are expressed in 
A
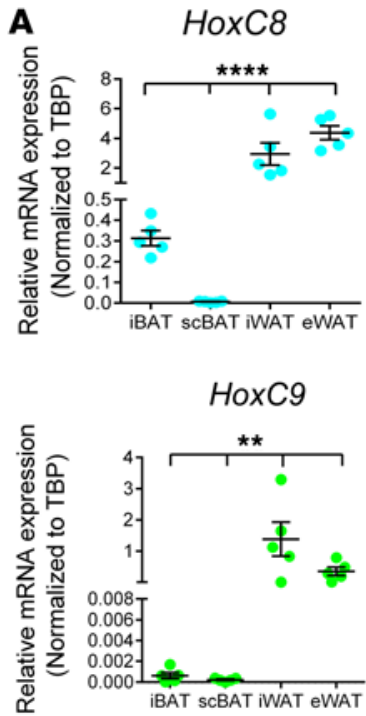

C

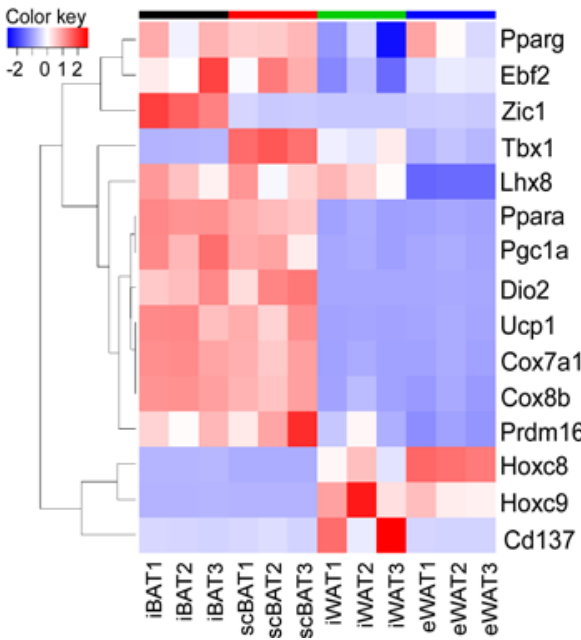

Zic 1

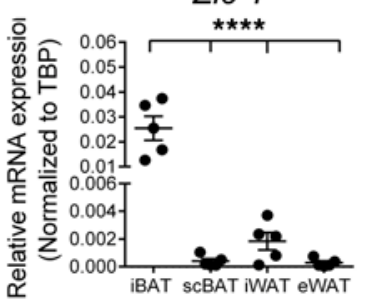

Lhx8

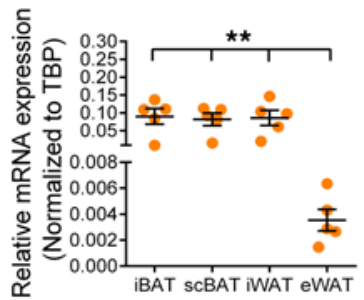

D

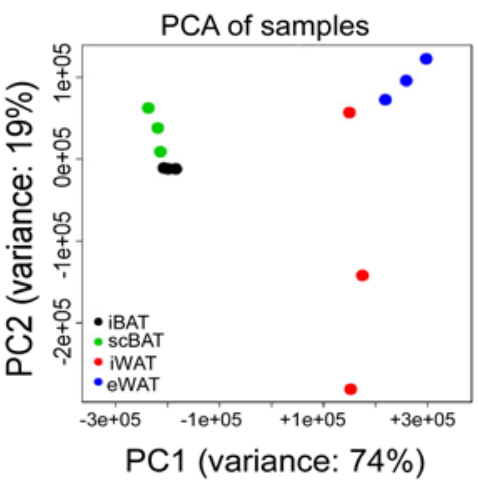

Tbx1

Cd137
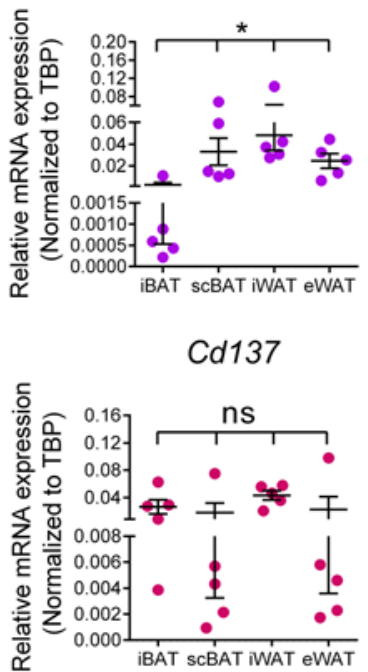

E

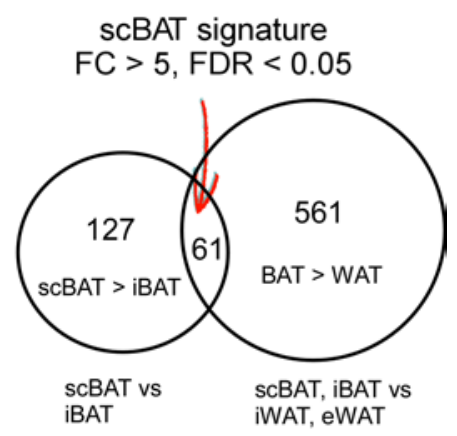

Figure 6. Genome-wide expression analysis identifies genes specifically expressed in scBAT. (A) Relative mRNA expression (qRT-PCR) of selective iBAT, WAT, or beige adipocyte markers in iBAT, scBAT, iWAT, and eWAT isolated from 8-week-old mice. Data are presented as mean \pm SEM. $n=5$. ${ }^{* * *} P<0.0001$, ${ }^{* * *} P<0.001,{ }^{* *} P<0.01,{ }^{*} P<0.05$; ns, nonsignificant $(P>0.05)$. One-way ANOVA. (B) Schematic showing the anatomical locations of iBAT, scBAT, iWAT, eWAT used for RNA-sequencing analyses. tr, trachea; $c b$, clavicle bone. (C) Clustered heat map of selected genes from the RNA-sequencing data in iBAT, scBAT, iWAT, and eWAT ( $n=3$ per tissue type). (D) Principal component analysis (PCA) of the RNA-sequencing samples. Samples were plotted in the first (PC1) and second (PC2) principal components. (E) Venn diagram showing the number of genes found to be specifically expressed in scBAT by RNA-sequencing. Sixty-one genes were significantly upregulated at least 5 -fold both in scBAT compared with iBAT and in BAT compared with WAT. FC, fold change.

human scBAT $(21,34), L h x 8$ (a proposed iBAT marker) and Tbxl (a proposed beige adipocyte marker), were also highly expressed in mouse scBAT by our qRT-PCR and RNA-sequencing studies (Figure 6, A and B). Together, our analyses indicate that genes from the molecular signature of human scBAT are also expressed in mouse scBAT, providing further evidence that mouse scBAT closely resembles human scBAT.

\section{Discussion}

Comprehensive studies led by S. Cinti's group identified several BAT depots in adult mice and provided a physiological blueprint for studying the function and metabolic contribution of mouse BAT (8). Here, we describe the existence of an additional BAT depot, scBAT, in mouse embryos and adult mice. Specifically, the mouse scBAT depot is situated above the clavicle bones in a region analogous to the region where human supraclavicular BAT resides. Like iBAT, mouse scBAT is densely packed with mitochondria, responds to $\beta 3$-adrenergic stimulation, and expresses Ucp1 at levels comparable to iBAT, suggesting that scBAT also 
A

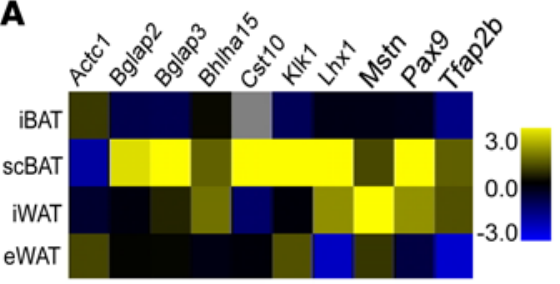

B

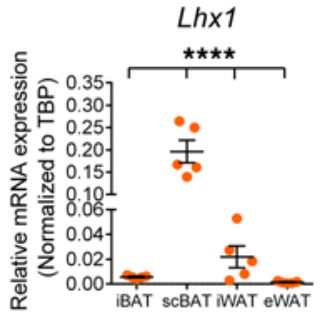

Bglap2

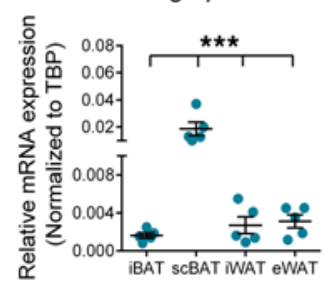

Cst10

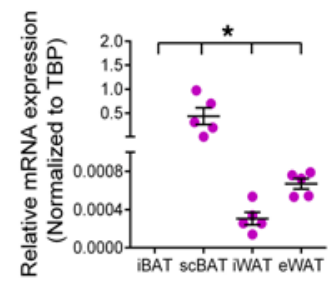

C

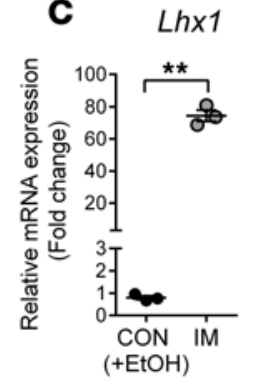

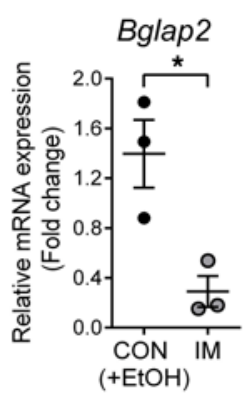

D

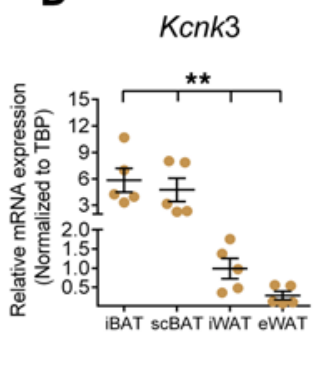

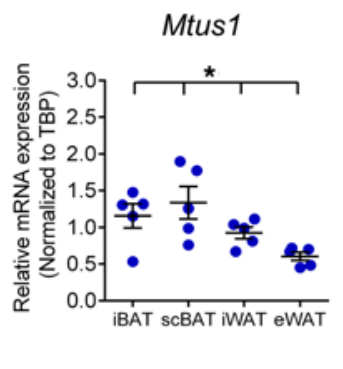

E

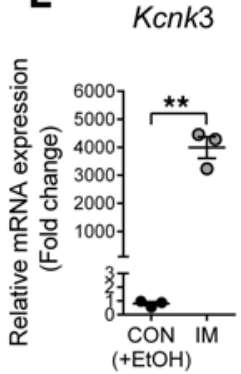

Mtus1

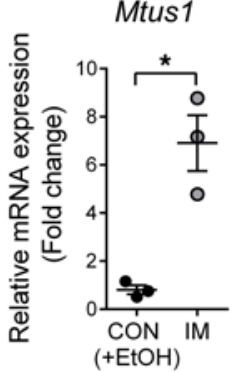

Figure 7. Validation of expression of novel and known genes in mouse scBAT. (A) Validation of RNA-sequencing results by qRT-PCR. Heat map of expression levels of 10 randomly selected genes from the 61 genes in Figure 6E in iBAT, scBAT, iWAT, and eWAT from 8-week-old mice. The heat map was constructed using the $\log _{2}$-transformed mean value of each tissue normalized to the median value of all samples in all 4 different adipose tissues. $n=5$. (B) Relative mRNA expression of $L h \times 1, B g l a p 2$, and Cst10 (Cst10 was absent in iBAT) in iBAT, scBAT, iWAT, and eWAT isolated from 8-week-old mice. Data are presented as mean \pm SEM. $n=5$. ${ }^{* * *} P<0.0001,{ }^{* *} P<0.001,{ }^{*} P<0.05$. One-way ANOVA. (C) Relative mRNA expression of $L h x 1$ and $B g l a p 2$ in differentiated P7scBAT-C4 cells. Data are presented as mean \pm SEM. $n=3$. ${ }^{*} P<0.01,{ }^{*} P<0.05$. Two-tailed $t$ test. CON, treated with vehicle (ethanol [EtOH]); $I M$, induction medium. (D) Relative mRNA expression of $K c n k 3$ and Mtus1 in iBAT, scBAT, iWAT, and eWAT. Data are presented as mean \pm SEM. $n=5$. ${ }^{* *} P$ $<0.01,{ }^{*} P<0.05$. One-way ANOVA. (E) Relative mRNA expression of $K c n k 3$ and Mtus1 in P7scBAT-C4 cells. Data are presented as mean \pm SEM. $n=3$. ${ }^{*} P<$ $0.01,{ }^{*} P<0.05$. Two-tailed $t$ test.

possesses the potential for thermoregulation. Adipose tissue transplantation studies further demonstrated that the effects of scBAT on glucose homeostasis, insulin sensitivity, and maintenance of body temperature under cold exposure are similar to those of iBAT. Interestingly, we also observed increased endogenous BAT mass in mice transplanted with either BBAT or scBAT. However, whether transplanted BAT can also regulate the metabolic properties of the endogenous BAT remains to be determined.

While these studies demonstrate that scBAT exhibits the potential for thermoregulation and glucose homeostasis, we hypothesize that the physiological contribution of scBAT may be different than that of iBAT and other BAT depots, such as subscapular BAT. Specifically, given that scBAT surrounds the jugular veins of the neck, the primary function of mouse scBAT could likely be to keep the blood vessels (veins) supplying circulation between the brain and heart warm, while iBAT and subscapular BAT keep the blood vessels responsible for circulation in the torso warm. In support of this notion, scBAT was also recently found near the jugular vein in humans (35). Together with previously identified deep neck BAT depots in the carotid sheath, where the common carotid artery and internal jugular vein are located (18), human neck BAT likely maintains the temperature of the circulatory system delivering blood to and from the brain.

As in previous reports, we observed that some of the markers for specific adipose depots in mice were not uniquely expressed in their proposed depots $(10,36-38)$. For example, $L h x 8$, a putative mouse iBAT marker, and Tbx1 and Cited1, two mouse beige adipocyte markers, were expressed in mouse iBAT, iWAT, and scBAT (Figure 6A) (10). To find genes specifically expressed in scBAT, we performed RNA-sequencing studies. While the gene expression profiles of scBAT and iBAT were very similar, a set of 61 genes was expressed at a higher level in scBAT than in iBAT and WAT. We verified by qRT-PCR that 3 of these genes, Lhx1, Bglap2, and Cst10, are specifically expressed in scBAT, suggesting that these genes could be good markers for scBAT. In addition, we found that expression of $L h x 1$ is higher in supraclavicular mature adipocytes, while expression of Bglap2 is higher in supraclavicular preadipocytes. This suggests that $L h x 1$, a transcription factor involved in tissue development (39-41), and Bglap2, a hormone secreted from the bone that can regulate insulin sensitivity (42), may have unique roles in the regulation of scBAT function. Further research is warranted to determine the roles of these newly identified scBAT-specific genes. 
Whether human scBAT is composed of classical BAT, beige adipoyctes, or a mixture of both is under debate $(11,17,21,22)$. Due to the lack of a BAT depot analogous to human scBAT, previous studies relied on using molecular markers proposed to be specific for iBAT or beige adipocytes to identify human BAT. To date, the expression of very few of these markers in human scBAT has been validated $(34,43)$. Two markers, $L h x 8$ and Tbx1, are expressed in human scBAT. Here, we also found that $L h \times 8$ and $T b x 1$ are also highly expressed in mouse scBAT. In addition, we found that two newly identified human scBAT markers, Kcnk3 and Mtus1, were also expressed in mouse scBAT. Kcnk3 is highly expressed in mouse iBAT $(44,45)$, in human scBAT and deep neck BAT $(26,35)$, and in mouse beige adipocytes (26), suggesting that $K c n k 3$, like $L h x 8$ and $T b x 1$, is not specifically expressed in one type of adipose tissue. Nevertheless, the observation that Kcnk3, Mtus1, Lhx8, and Tbx1 are all expressed in mouse scBAT provides molecular evidence that mouse scBAT is similar to human scBAT. These observations also suggest that the controversy regarding the identity of human scBAT may be due to the specificity of the molecular markers used in previous studies and the lack of an animal model for human scBAT. Given that mouse scBAT is more representative of human scBAT than the depots used previously, future studies using mouse scBAT should provide a more specific molecular signature for human scBAT.

Morphologically, adult human scBAT is composed of a mixture of adipocytes that contain either a single large lipid droplet or multiple small lipid droplets, while mouse scBAT is more uniformly composed of adipocytes with multiple small lipid droplets. This morphological discrepancy is likely due to differences in living conditions; for example, humans maintain thermoneutrality so BAT is not activated, while mice are typically housed at subthermoneutrality so BAT is activated. Other factors, such as diet composition, age, disease status, and genetic factors, may also contribute to this difference. Given the similarities between mouse and human scBAT, such studies should also allow us to better understand how human scBAT functions and how environmental influences affect its activity and to test new drug targets in mice.

Finally, while our studies indicate that scBAT is classical BAT because the depot is formed during embryogenesis and is morphologically and thermogenically similar to iBAT, we cannot rule out the possibility that human scBAT may share some characteristics with mouse beige adipocytes, as reported by Shinoda et al. In their studies, they found that the expression profiles of human scBAT adipocytes clustered more closely with those of beige adipocytes than with iBAT adipocytes after stimulation with standard adipogenic induction media and rosiglitazone (26). In this study, we used adipose tissues for RNA-sequencing analysis. Our sequencing data, therefore, capture the transcriptional landscape of an adipose tissue in a more natural, unstimulated state. It is difficult to assess how the in vitro treatment conditions in studies using cultured cells may have influenced gene expression. Future studies that compare supraclavicular adipocytes with beige adipocytes and human supraclavicular adipocytes under the same adipogenic treatment conditions should provide further clarification of this issue.

Here we provide data indicating the discovery of a new BAT depot in mice that is analogous to human scBAT. Our studies show that mouse scBAT is a classical BAT depot that shares molecular markers with human scBAT and provide an animal model for studying human scBAT function. Future studies involving mouse scBAT should allow us to explore more translatable approaches for utilizing BAT to treat health conditions associated with obesity and its related complications.

\section{Methods}

Complete experimental procedures are included in Supplemental Methods.

Animals. All mouse experiments (except adipose tissue transplantation experiments) involved male C57BL/ 6 mice acquired from the Center for Comparative Medicine Production Colony at Baylor College of Medicine (BCM). For adipose tissue transplantation experiments, 12-week-old male C57BL/6 mice from Charles River Laboratories were used as donor and recipient mice for transplantation at the Ohio State University (OSU).

Embryo processing and histology. For histological analysis, mouse embryos were collected, fixed in 4\% paraformaldehyde, and embedded in paraffin. These embryos were sectioned at $6-\mu \mathrm{m}$ thickness and stained with $\mathrm{H} \& \mathrm{E}$ as previously described (27).

Isolation of adipose tissues. iBAT, iWAT, and eWAT were isolated as previously described (10). To isolate scBAT, we first opened the mouse ventral neck by making a skin incision from the sternum to the lower jaw with a surgical scissor at the ventral midline. We further opened the skin incision laterally to reveal the ventral neck structures, including the subcutaneous WAT, the superficial muscle layer, and the 
salivary gland. We then cut off the superficial muscle layer and moved the salivary gland upward with a pair of forceps to expose the inner neck structures, including the scBAT, jugular veins, sternocleidomastoid muscles, and trachea (covered by the sternohyoid muscle) under the dissecting microscope. scBAT was then dissected free from the connecting veins using a pair of fine forceps. After thoroughly removal of any non-adipose tissues from iBAT, scBAT, iWAT, and eWAT, the weights were recorded. For gene expression analyses of scBAT and iBAT from CL316243-treated mice, mice received $1 \mathrm{mg} / \mathrm{kg}$ by i.p. injection daily for 3 days before adipose tissues were collected 24 hours after the final injection.

Lipid droplet size measurement. H\&E-stained adipose tissue sections from 8-week-old mice were imaged at $400 \times$ magnification using a DS-Fil camera connected to a Nikon Eclipse 80i stereomicroscope. Lipid droplet size in the center area $(70 \mu \mathrm{m} \times 70 \mu \mathrm{m})$ of each image was measured using the area measurement tool of Nikon NIS Elements AR 3.2 software. Four to eight adipose tissue sections were randomly selected for the measurement.

$R N A$ isolation and $q R T-P C R$. Frozen adipose tissues were homogenized and total RNA was extracted using an Aurum Total RNA Fatty and Fibrous Tissue Kit following the manufacturer's instructions (BioRad). Two micrograms of total RNA was used to synthesize first-strand cDNA using the SuperScript III First-Strand Synthesis System (Invitrogen). qRT-PCR reactions were performed using Platinum SYBR Green qPCR SuperMix-UDG (Invitrogen) in a CFX96 Touch Real-Time PCR Detection System (Bio$\mathrm{Rad})$. The $\Delta \mathrm{Ct}$ method (2- $\left.2^{-\Delta \mathrm{Ct}}\right)$ was used to calculate the relative mRNA expression level of each gene. Specific gene expression was normalized to either $\beta$-actin or TATA box binding protein (TBP). Primers used for qRT-PCR are listed in Supplemental Table 3.

Protein isolation and Western blotting. Western blotting was performed as previously described (46). Procedures and primary and secondary antibodies used in this study are provided in Supplemental Methods.

$R N A$-sequencing and data analysis. Total RNA was isolated from scBAT, iBAT, iWAT, and eWAT as described above. Total RNA from each adipose tissue sample was sent to the BCM Genomic and RNA profiling Core for RNA-sequencing library preparation and sequencing using a HiSeq 2500 instrument (Illumina). RNA sequencing reads were mapped to the mouse genome (mm10) using STAR with NCBI RefSeq genes as the reference. EdgeR (http://bioconductor.org/packages/release/bioc/html/edgeR.html) was used to analyze the gene-based read counts to detect differentially expressed genes between different adipose tissue samples. The FDR of the differentially expressed genes was estimated using the method of Benjamini and Hochberg. An FDR less than 0.05 was considered statistically significant.

TEM. TEM experiments were conducted by the BCM Integrated Microscopy Core. Detailed experimental procedures are provided in Supplemental Methods.

BAT transplantation studies. Transplantation was performed using BAT removed from the interscapular (iBAT) or supraclavicular (scBAT) region of 12-week-old male C57BL/6 mice and transplanted ( $0.1 \mathrm{~g}$ of iBAT and scBAT) into the visceral cavity of the recipient (29). Mice that were sham-operated underwent the same procedure, but instead of transplanting BAT, we located, exposed, and then replaced their epididymal fat pads. GTTs and ITTs were performed in BAT-transplanted and sham-operated mice. GTTs were performed every 2 weeks after transplantation. Mice were fasted for 11 hours and received i.p. glucose $(2.0 \mathrm{~g} / \mathrm{kg}$ body weight). ITTs were performed at 12 weeks after transplantation. Mice were fasted for 2 hours and received i.p. insulin (1 U/kg body weight) (Humulin; Eli Lilly). Blood glucose concentrations were determined using a OneTouch Ultra portable glucometer (LifeScan).

Core body temperature measurement. For BAT-transplanted mice, mice were fasted overnight, and body temperature was determined using an animal rectal probe connected to a $\mathrm{TH}-8$ thermometer (Physitemp). Basal temperature was determined; animals were then housed at $4^{\circ} \mathrm{C}$, and body temperature was measured at 15, 30, 60, 90, and 120 minutes.

Statistics. The 2-tailed $t$ test was used to evaluate statistical significance between 2 groups. Oneway ANOVA was used to evaluate statistical significance between 3 or more groups. A $P$ value less than 0.05 was considered statistically significant. Statistical analyses were performed using GraphPad Prism 6 software.

Accession numbers. The NCBI Gene Expression Omnibus (GEO) number for RNA-sequencing data reported here is GEO GSE92844.

Study approval. All animal experiments were approved by the Institutional Animal Care and Use Committees of BCM and the OSU. 


\section{Author contributions}

MHC and KIS designed the experiments. MHC, QM, JS, TR, LAB, FJM, KIS, EJJ, ACL, XG, and QT conducted research and analyzed data; MHC wrote the manuscript; KIS, EJJ, and YHT edited the manuscript; AMNA, YHT, and FS consulted on the study and provided important reagents.

\section{Acknowledgments}

The authors wish to thank Yuxiang Sun for useful advice and Chitra Jeyasankar for technical support. This study was supported by USDA/ARS CRIS 3092-5-001-059, NIH P30-DK079638, and American Heart Association 16GRNT30720003 to MHC; and NIH K01-DK105109 to KIS. RNA sequencing and data analyses were supported in part by the Genomic and RNA Profiling Core at BCM with funding from the NIH Center grant (P30-DK079638). TEM analyses were supported by the Integrated Microscopy Core at BCM with funding from the NIH (HD007495, DK56338, and CA125123).

Address correspondence to: Miao-Hsueh Chen, Department of Pediatrics, Baylor College of Medicine, One Baylor Plaza, MS:BCM320, Houston, Texas 77030, USA. Phone:713.798.0357; E-mail:miaohsuc@bcm.edu.

1. Ogden CL, Carroll MD, Fryar CD, Flegal KM. Prevalence of obesity among adults youth: United States, 2011-2014. NCHS Data Brief. 2015;(219):1-8.

2. Cinti S, Morroni M. Brown adipocyte precursor cells: a morphological study. Ital J Anat Embryol. 1995;100(Suppl 1):75-81.

3. Kajimura S, Saito M. A new era in brown adipose tissue biology: molecular control of brown fat development and energy homeostasis. Annu Rev Physiol. 2014;76:225-249.

4. Gesta S, Tseng YH, Kahn CR. Developmental origin of fat: tracking obesity to its source. Cell. 2007;131(2):242-256.

5. Holm C, Fredrikson G, Cannon B, Belfrage P. Hormone-sensitive lipase in brown adipose tissue: identification and effect of cold exposure. Biosci Rep. 1987;7(11):897-904.

6. Bartelt A, et al. Brown adipose tissue activity controls triglyceride clearance. Nat Med. 2011;17(2):200-205.

7. Cannon B, Nedergaard J. Brown adipose tissue: function and physiological significance. Physiol Rev. 2004;84(1):277-359.

8. Frontini A, Cinti S. Distribution and development of brown adipocytes in the murine and human adipose organ. Cell Metab. 2010;11(4):253-256.

9. Vitali A, Murano I, Zingaretti MC, Frontini A, Ricquier D, Cinti S. The adipose organ of obesity-prone C57BL/6J mice is composed of mixed white and brown adipocytes. J Lipid Res. 2012;53(4):619-629.

10. de Jong JM, Larsson O, Cannon B, Nedergaard J. A stringent validation of mouse adipose tissue identity markers. Am J Physiol Endocrinol Metab. 2015;308(12):E1085-E1105.

11. Wu J, et al. Beige adipocytes are a distinct type of thermogenic fat cell in mouse and human. Cell. 2012;150(2):366-376.

12. Petrovic N, Walden TB, Shabalina IG, Timmons JA, Cannon B, Nedergaard J. Chronic peroxisome proliferator-activated receptor gamma (PPARgamma) activation of epididymally derived white adipocyte cultures reveals a population of thermogenically competent, UCP1-containing adipocytes molecularly distinct from classic brown adipocytes. J Biol Chem. 2010;285(10):7153-7164.

13. Cypess AM, et al. Identification and importance of brown adipose tissue in adult humans. N Engl J Med. 2009;360(15):1509-1517.

14. van Marken Lichtenbelt WD, et al. Cold-activated brown adipose tissue in healthy men. N Engl J Med. 2009;360(15):1500-1508.

15. Virtanen KA, et al. Functional brown adipose tissue in healthy adults. N Engl J Med. 2009;360(15):1518-1525.

16. Saito M, et al. High incidence of metabolically active brown adipose tissue in healthy adult humans: effects of cold exposure and adiposity. Diabetes. 2009;58(7):1526-1531.

17. Zingaretti MC, et al. The presence of UCP1 demonstrates that metabolically active adipose tissue in the neck of adult humans truly represents brown adipose tissue. FASEB J. 2009;23(9):3113-3120.

18. Cypess AM, et al. Anatomical localization, gene expression profiling and functional characterization of adult human neck brown fat. Nat Med. 2013;19(5):635-639.

19. Xue R, et al. Clonal analyses and gene profiling identify genetic biomarkers of the thermogenic potential of human brown and white preadipocytes. Nat Med. 2015;21(7):760-768.

20. Chondronikola M, et al. A percutaneous needle biopsy technique for sampling the supraclavicular brown adipose tissue depot of humans. Int J Obes (Lond). 2015;39(10):1561-1564.

21. Jespersen NZ, et al. A classical brown adipose tissue mRNA signature partly overlaps with brite in the supraclavicular region of adult humans. Cell Metab. 2013;17(5):798-805.

22. Lidell ME, et al. Evidence for two types of brown adipose tissue in humans. Nat Med. 2013;19(5):631-634.

23. Chondronikola M, et al. Brown adipose tissue improves whole-body glucose homeostasis and insulin sensitivity in humans. Diabetes. 2014;63(12):4089-4099.

24. Chondronikola M, et al. Brown adipose tissue activation is linked to distinct systemic effects on lipid metabolism in humans. Cell Metab. 2016;23(6):1200-1206.

25. Cypess AM, et al. Activation of human brown adipose tissue by a $\beta 3$-adrenergic receptor agonist. Cell Metab. 2015;21(1):33-38.

26. Shinoda K, et al. Genetic and functional characterization of clonally derived adult human brown adipocytes. Nat Med. 2015;21(4):389-394.

27. Nosavanh L, Yu DH, Jaehnig EJ, Tong Q, Shen L, Chen MH. Cell-autonomous activation of Hedgehog signaling inhibits brown adipose tissue development. Proc Natl Acad Sci USA. 2015;112(16):5069-5074.

28. Cohen P, et al. Ablation of PRDM16 and beige adipose causes metabolic dysfunction and a subcutaneous to visceral fat switch. Cell. 2014;156(1-2):304-316. 
29. Stanford KI, et al. Brown adipose tissue regulates glucose homeostasis and insulin sensitivity. J Clin Invest. 2013;123(1):215-223.

30. Liu X, et al. Brown adipose tissue transplantation reverses obesity in ob/ob mice. Endocrinology. 2015;156(7):2461-2469.

31. Gesta S, et al. Evidence for a role of developmental genes in the origin of obesity and body fat distribution. Proc Natl Acad Sci USA. 2006;103(17):6676-6681.

32. Timmons JA, et al. Myogenic gene expression signature establishes that brown and white adipocytes originate from distinct cell lineages. Proc Natl Acad Sci USA. 2007;104(11):4401-4406.

33. Sharp LZ, et al. Human BAT possesses molecular signatures that resemble beige/brite cells. PLoS ONE. $2012 ; 7(11)$ :e49452.

34. Lidell ME, Betz MJ, Enerback S. Two types of brown adipose tissue in humans. Adipocyte. 2014;3(1):63-66.

35. Porter C, et al. Human and mouse brown adipose tissue mitochondria have comparable UCP1 function. Cell Metab. 2016;24(2):246-255

36. Long JZ, et al. A smooth muscle-like origin for beige adipocytes. Cell Metab. 2014;19(5):810-820.

37. Garcia RA, Roemmich JN, Claycombe KJ. Evaluation of markers of beige adipocytes in white adipose tissue of the mouse. Nutr Metab (Lond). 2016;13:24

38. Nakhuda A, et al. Biomarkers of browning of white adipose tissue and their regulation during exercise- and diet-induced weight loss. Am J Clin Nutr. 2016;104(3):557-565.

39. Shawlot W, Behringer RR. Requirement for Lim1 in head-organizer function. Nature. 1995;374(6521):425-430

40. Kobayashi A, Kwan KM, Carroll TJ, McMahon AP, Mendelsohn CL, Behringer RR. Distinct and sequential tissue-specific activities of the LIM-class homeobox gene Lim1 for tubular morphogenesis during kidney development. Development. 2005;132(12):2809-2823.

41. Bedont JL, et al. Lhx1 controls terminal differentiation and circadian function of the suprachiasmatic nucleus. Cell Rep. 2014;7(3):609-622.

42. Lee NK, et al. Endocrine regulation of energy metabolism by the skeleton. Cell. 2007;130(3):456-469.

43. Scheele C, Larsen TJ, Nielsen S. Novel nuances of human brown fat. Adipocyte. 2014;3(1):54-57.

44. Pisani DF, et al. The $\mathrm{K}+$ channel TASK1 modulates $\beta$-adrenergic response in brown adipose tissue through the mineralocorticoid receptor pathway. FASEB J. 2016;30(2):909-922.

45. Svensson PA, et al. Gene expression in human brown adipose tissue. Int J Mol Med. 2011;27(2):227-232.

46. Chen MH, et al. Cilium-independent regulation of Gli protein function by Sufu in Hedgehog signaling is evolutionarily conserved. Genes Dev. 2009;23(16):1910-1928. 